\title{
SpanAlign: Efficient Sequence Tagging Annotation Projection into Translated Data applied to Cross-Lingual Opinion Mining
}

\author{
Léo Jacqmin $^{1}$ Gabriel Marzinotto ${ }^{1}$ Justyna Gromada ${ }^{2}$
}

\section{Ewelina Szczekocka $^{2}$ Robert Kołodyński ${ }^{2}$ Antoine Chauvière ${ }^{1}$ Géraldine Damnati $^{1}$}

\author{
(1) Orange Innovation / Lannion, France \\ (2) Orange Innovation Poland / Warsaw, Poland \\ first. lasteorange.com
}

\begin{abstract}
Following the increasing performance of neural machine translation systems, the paradigm of using automatically translated data for cross-lingual adaptation is now studied in several applicative domains. The capacity to accurately project annotations remains however an issue for sequence tagging tasks where annotation must be projected with correct spans. Additionally, when the task implies noisy usergenerated text, the quality of translation and annotation projection can be affected. In this paper we propose to tackle multilingual sequence tagging with a new span alignment method and apply it to opinion target extraction from customer reviews. We show that provided suitable heuristics, translated data with automatic span-level annotation projection can yield improvements both for crosslingual adaptation compared to zero-shot transfer, and data augmentation compared to a multilingual baseline.
\end{abstract}

\section{Introduction}

Large self-supervised pre-trained models which are fine-tuned on downstream tasks have become the de facto standard in NLP. However, monolingual pre-trained models are only available in some high resource languages due to data limitations. Additionally, in multilingual settings, having a separate model for each language quickly becomes unpractical. Multilingual models which are pretrained on monolingual corpora in multiple languages such as multilingual BERT (mBERT) and XLM-R provide an alternative. They have been shown to yield on par, if not better performance on various tasks compared to monolingual models and perform particularly well for low-resource languages provided similar languages are represented in the training data (Conneau et al., 2020). What's more, they have shown surprising capacities for generalization with zero-shot cross-lingual transfer, in which a model is fine-tuned for a task using annota- ted data in a specific language and then evaluated in an unseen language (Radford et al., 2019). Considering that labeled data is more readily available for certain languages, this technique of cross-lingual transfer can be harnessed to process low-resource languages, therefore bypassing the need for a costly annotation process. Some research effort has been done in grasping to what extent these models are language agnostic (Pires et al., 2019), though more probing is necessary in order to fully understand and measure how multilingual these models are.

Another approach to cross-lingual transfer consists in using machine translation (MT) to adapt the annotated training data available in a source language to the target language. The resulting translated data is used to train a model with supervision in the target language. Producing a label for translated data is straightforward for sentence-level tasks. However, little work focuses on adapting datasets annotated at the word level. Such adaptation requires an additional step of label projection which can be error-prone and introduce noise in the training data. A common way of projecting labels consists in obtaining word alignments between source and target utterances and then projecting the labels. In a recent paper, (Li et al., 2020) argued that zero-shot cross-lingual transfer surpassed translation-based adaptations for several sequence tagging tasks, and proposed to first warm up the model's weights on the translated data only and then fine-tuning on the original data. In contrast, we show that translationbased adaptation yields superior performance compared to zero-shot cross-lingual transfer, provided the right annotation projection method is used.

We propose to apply this method in the context of opinion mining, and specifically to aspect-based sentiment analysis (ABSA) (Pontiki et al., 2014). Nowadays, people increasingly rely on reviews and comments, e.g. on social media and review websites, to select which products to buy or which services to use. Companies can also make use of these 
data which provide insightful feedback from their customers. ABSA is concerned with extracting finegrained information from customer feedback, e.g. which aspects of a product or a service are being referred to, which entities are associated with them, and what is the customer's attitude towards them. One challenge that this task poses is that reviews are user-generated and can thus be very noisy. Online users tend to produce text that derives from the standard form of language with e.g. misspellings, internet slang, abbreviations, phonetic transcriptions, and missing or incorrect punctuation marks. For companies operating in several countries, another challenging aspect is the multiplicity of languages found in these reviews. Accordingly, multilingual approaches to opinion mining are essential to efficiently analyze customer feedback across countries.

We first review previous works related to crosslingual transfer and ABSA. We then present our translation-based adaptation approach, and show its relevance in two scenarios : cross-lingual adaptation where annotated data is only available in the source language, and data augmentation where annotated data is available for both source and target languages. Lastly, we turn to an analysis of noisy user-generated text and propose a heuristic to filter out noisy utterances before translation.

\section{Related Work}

\subsection{Aspect-based Sentiment Analysis}

Due to the difficulties associated with processing user-generated text, opinion mining is commonly formulated as a text classification task concerned with e.g. classifying the overall polarity of a sentence by assigning it a polarity label (Pang and Lee, 2008).

Aspect-based sentiment analysis (ABSA) (Hu and Liu, 2004; Popescu and Etzioni, 2005; Pontiki et al., 2014, 2015, 2016) is a more fine-grained opinion mining task with several sub-tasks associated with it. For example, opinion target extraction (OTE) retrieves the entity on which an opinion is expressed in a review, and aspect sentiment classification (ASC) identifies the polarity of an opinion expressed on a given target entity. Some works focus on one subtask at a time, e.g. OTE (Li and Lam, 2017; Xu et al., 2018) or opinion word extraction (OWE) (Fan et al., 2019; Pouran Ben Veyseh et al., 2020). But this division of subtasks is ill-suited for real-world scenarios as both ASC and OWE assume that the opinion target is given. Moreover, these subtasks aim to extract related information. To facilitate practical applications of ABSA, recent works address OTE and ASC simultaneously ( $\mathrm{Li}$ et al., 2019a,b). The problem can be formulated as a sequence tagging task with unified labels to simultaneously detect opinion targets and the corresponding aspect sentiments. Some works went even further by addressing OTE, ASC, and OWE simultaneously, a task dubbed as aspect sentiment triplet extraction (Peng et al., 2020; Wang et al., 2021).

Most works in the state of the art focus on SemEval datasets from 2014 to 2016, with data from the restaurant, hotel or laptop domain in English. However, SemEval data are also available in several languages. Jebbara and Cimiano (2019), for instance, evaluated CNN models for OTE with multilingual word embeddings in a zero-shot crosslingual framework.

\subsection{Cross-lingual transfer}

Multilingual pre-trained models have shown strong capacities for generalization and have been successfully applied for zero-shot cross-lingual transfer on a variety of natural language understanding tasks (Wu and Dredze, 2019). This enables the application of such models to low-resource languages for which little or no labelled data is available.

Machine Translation (MT) can help learning cross-lingual representations and transfering information across languages. Rather than being at odds, zero-shot cross-lingual transfer and MT-based approaches are complementary : MT can be used to generate synthetic data in languages for which no annotated data is available. Accordingly, MT can improve cross-lingual transfer in two ways : (i) By translating the training data into the target languages and fine-tuning on all languages, e.g. for subjectivity analysis (Banea et al., 2008), sentiment classification (Duh et al., 2011) or semantic role labeling (Fei et al., 2020). (ii) Or by applying a model fine-tuned on the source language to a test set translated from target to source language. (Conneau et al., 2020) refers to the former approach as translate-train and to the latter as translate-test. In this work, we focus on the former approach. This approach naturally applies to sentence classification tasks and previous work has shown that translation-based adaptation is superior to zero- 
shot cross-lingual transfer for text classification (Schwenk and $\mathrm{Li}, 2018$ ) and text pair classification (Conneau et al., 2018; Yang et al., 2019). However, little work focuses on adapting datasets annotated at the word level for sequence tagging. This requires an important additional step of annotation projection as no word-to-word correspondence is available.

One approach to annotation projection for sequence tagging tasks relies on obtaining word alignments to project labels from source to target utterances. Several statistical word alignment tools are available such as fast_align (Dyer et al., 2013) which constitutes the usual baseline for this approach. Starting from the assumption that neural machine translation (NMT) models capture word alignment through their attention mechanism, other works (Chen et al., 2020; Zouhar and Pylypenko, 2021) focus on using attention weights for word alignment. In an attempt to alleviate the need for parallel corpora, (Jalili Sabet et al., 2020) leveraged modern multilingual pre-trained models and released SimAlign, a tool for unsupervised word alignment based on the similarity of multilingual word representations.

In order to project sequence annotations, one has to leverage word-level alignment towards span alignment. Marzinotto (2020) used attention-based word alignments to project FrameNet annotations (targets and Frame Elements) into a target language. Others have sought to improve label projection through different approaches. Jain et al. (2019) proposed an entity projection method for named entity recognition, in which they obtain potential translations of an entity in the target language and select the best match with the source entity. To make use of task-related information, Xu et al. (2020) proposed an end-to-end model to jointly align and predict target slot labels for cross-lingual NLU. (Li et al., 2020) propose an approach called span-tospan mapping which derives span alignment from word alignments. They applied it to several tasks (Opinion Mining, Semantic Role Labeling, Named Entities) but didn't obtain satisfactory results when directly using the translated and aligned data to fine-tune their models.

\section{SpanAlign}

In the case of sentence-level tasks, the translated data can be used right away using the same labels as in the source language corpus. In contrast,

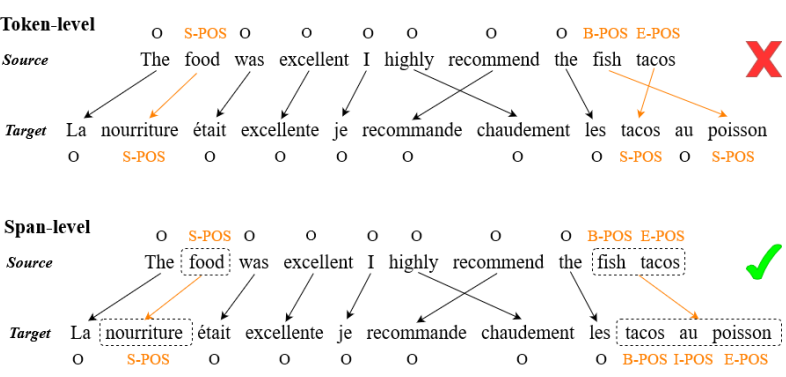

FIGURE 1: Different strategies for annotation projection using word alignments.

adapting sequence tagging datasets annotated at the word-level requires an additional step of annotation projection. A word-to-word correspondence between source and target utterances can be obtained using word alignment tools. One simple approach to annotation projection is then to project the gold labels in the source utterance to their corresponding word in the target utterance using this mapping. However, as token-level annotations may span several words, this approach can be error-prone, as illustrated in Figure 1. Consequently, we introduce a span-based alignment approach to project gold annotations at the span level. Additionally we make the assumption that translated data with erroneous annotation alignment are more likely to be harmful for the subsequent training process, and propose to introduce a constraint on span alignment to filter out translated annotations and remove them from the candidate pool of translated training data.

\subsection{Annotation Projection for Sequence Tagging}

Let $x_{1: N}^{s r c}=\left\{x_{1}, \ldots, x_{N}\right\}$ be the source utterance and $x_{1: M}^{\text {trg }}=\left\{x_{1}, \ldots, x_{M}\right\}$ the translated utterance. We obtain word alignments $a_{1: N}^{s r c}=\left\{a_{1}, \ldots, a_{N}\right\}$ from these paired utterances, with $a_{i} \in[1, M]$ $\cup\{$ NULL $\}$ indicating which words in the target utterance correspond to source word $x_{i}^{s r c}$. For each annotated span $x_{i: j}^{s r c}$ in the source utterance from the $i$-th source word to the $j$-th source word, we identify the projected span in the target utterance as $x_{p: q}^{t r g}$, where

$$
\begin{aligned}
& p=\min \left(a_{i j}^{s r c}\right) \\
& q=\max \left(a_{i j}^{s r c}\right)
\end{aligned}
$$

This procedure is described in pseudo-code in Algorithm 1. We then assign the label without position, e.g. POS, to all words in the projected span and reformat the position labels, e.g. $B-\{P O S\}$, $\mathrm{E}-\{\mathrm{POS}\}$. 


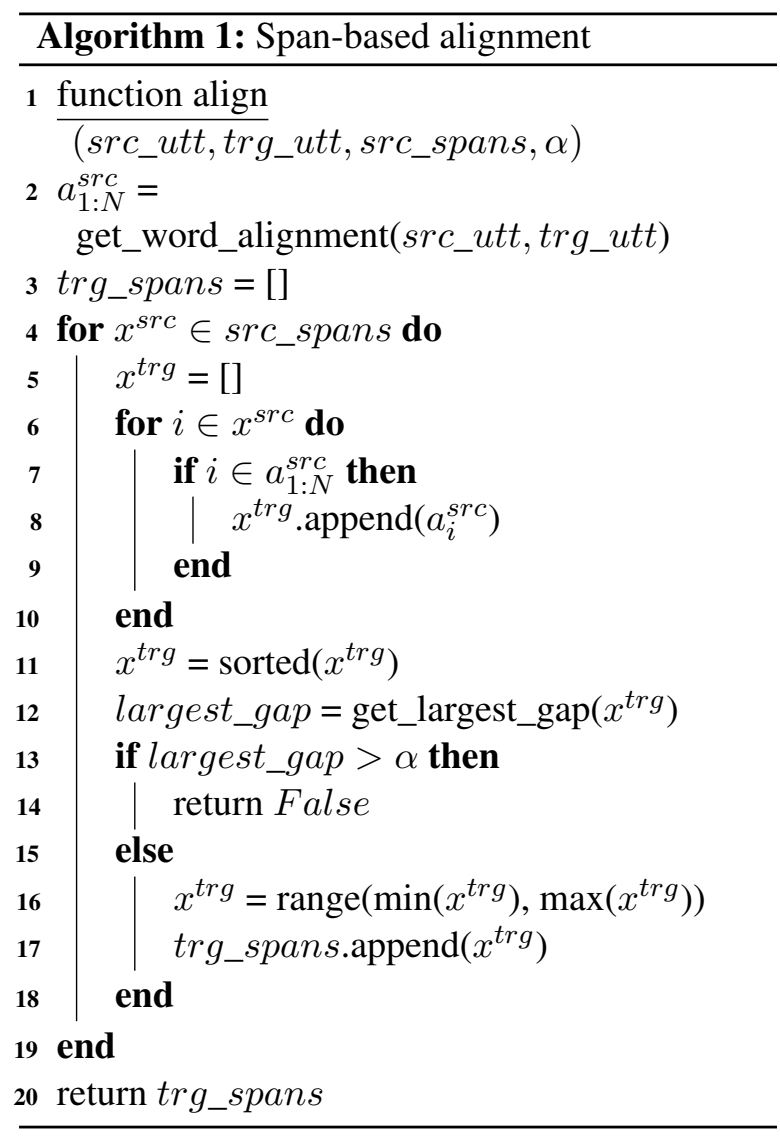

\subsection{Filtering Ill-formed Projected Spans}

The underlying word alignments are not guaranteed to be entirely accurate. First, the translations are machine-generated and can therefore be inadequate. Second, user-generated text is noisy. This noise in source utterances can negatively impact the translations and let errors propagate through the rest of the pipeline. To address these issues, we use a heuristic to filter out pseudo-labeled utterances that are likely to be ill-formed. See Figure 2 for an example.

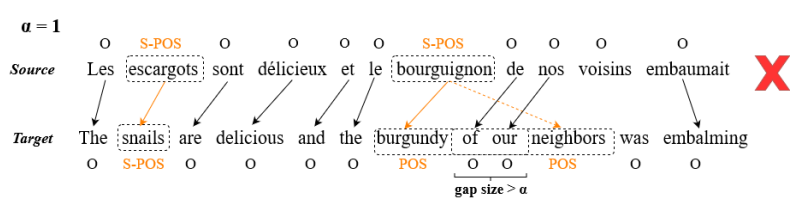

FIGURE 2: In this case, "bourguignon" which is a dish but also a gentilic was aligned with both "burgundy" and "neighbours", introducing a gap of two words in the proposed translation.

Since our projection method uses the minimum and maximum indices of the aligned words as the offset of the projected span, some gaps can appear in the projected spans where some of the translated words have not been aligned to an opinion target word in the source utterance. We call these words insertions. The hyperparameter $\alpha$ corresponds to the number of allowed insertions within a gap in the projected spans. Utterances that contain a projected span with a gap containing a number of insertions strictly higher than $\alpha$ are considered ill-formed and are filtered out of the translated dataset. Note that this heuristic is well-suited for the task of opinion target extraction because opinion targets are mostly adjacent nominal groups that usually translate into adjacent groups. It should also be relevant for tasks such as Named Entity detection. We are aware that some grammatical structures can translate into non adjacent terms in some languages but we believe that our heuristic approach is reasonable for entity detection tasks.

\section{Translation-based Adaptation}

Modern neural machine translation (NMT) provides satisfactory results thanks to Transformer models and has become increasingly available. As a result, such models can be used effectively to create synthetic data by translating a source language reference corpus.

We use Marian NMT ${ }^{1}$ to translate the source language corpora into the other languages, and viceversa. Marian NMT is open source and allows the use of pre-trained NMT models from the OPUSMT project (Tiedemann and Thottingal, 2020) available on this framework. A description of each model and its evaluation on benchmarks can be found online. ${ }^{2}$ To obtain word alignments, we use both off-the-shelf fast_align (Dyer et al., 2013) and SimAlign (Jalili Sabet et al., 2020). For the latter, which doesn't need any parallel data for training but relies on multilingual word representations, we use the Itermax variant, which was shown to outperform other word alignment approaches. Note that we did not adapt the translation models nor the alignment approaches to our specific domains.

\subsection{Cross-lingual Adaptation}

Cross-lingual adaptation denotes the case where annotated data is available for one language only and we wish to process other languages. The question here is whether the original annotated data is sufficient to fine-tune a pre-trained multilingual model and process unseen languages, or if translating this data into the other languages facilitates

1. https://marian-nmt github.io

2. https://opus.nlpl.eu/Opus-MT/ 
cross-lingual transfer. In other words, whether multilingual word representations are truly language agnostic or not. We experiment with the following experiments. $\mathbf{O}$ and $\mathbf{T r}$ respectively denote original and translated data. $\mathbf{S}$ and $\mathbf{T}$ refer to source and target languages.

$\mathbf{O}_{S}$ (Zero-shot) In the context of cross-lingual transfer, zero-shot learning consists in fine-tuning a model for a given task using annotated data in a source language, and then evaluating it for the same task on a test set from an unseen target language. This configuration serves as the baseline method for cross-lingual adaptation.

$\operatorname{Tr}_{S \rightarrow T}$ The first alternative configuration we consider is to simply adapt the available annotated data to the target language. For a given target language, we fine-tune the model on the translation of the source language data into the target language only.

$\mathbf{O}_{S}+\operatorname{Tr}_{S \rightarrow T} \quad$ As a step up from the previous configuration, we fine-tune a model on the concatenation of the source data and its translation into the target language.

$\mathbf{O}_{S}+\operatorname{Tr}_{S \rightarrow \text { all }} \quad$ In this last configuration, we use the concatenation of the source data with its translations into all the other languages. This approach results in a single model that can be applied to all languages.

\begin{tabular}{lccc}
\hline & Original & \multicolumn{2}{c}{ Translated } \\
\hline Config. & Source & Target & Others \\
\hline $\mathbf{O}_{S}$ (Zero-shot) & $\checkmark$ & & \\
$\mathbf{T r}_{S \rightarrow T}$ & & $\checkmark$ & \\
$\mathbf{O}_{S}+\mathbf{T r}_{S \rightarrow T}$ & $\checkmark$ & $\checkmark$ & \\
$\mathbf{O}_{S}+\mathbf{T r}_{S \rightarrow \text { all }}$ & $\checkmark$ & $\checkmark$ & $\checkmark$ \\
\hline
\end{tabular}

TABLE 1: Data configurations for cross-lingual adaptation.

\subsection{Data Augmentation}

Data augmentation refers to the case where small amounts of annotated data is available in all the languages we wish to process. We are interested in studying how additional synthetic data will affect the model's performance. We experiment with the following configurations : ${ }^{3}$

3. Note that not all possible configurations were reported for the sake of simplicity.
$\mathbf{O}_{T}$ (Monolingual) As a baseline configuration, we simply fine-tune a model on the original data for a given language and evaluate it on that language.

$\mathbf{O}_{\text {all }}$ (Multilingual) The next configuration uses the concatenation of the original data available in all the languages.

$\mathbf{O}_{\text {all }}+\operatorname{Tr}_{S \rightarrow \text { all }}$ We fine-tune a model on the concatenation of the original data in all languages along with the translated data from the source language into the other languages. With this configuration, we evaluate the relevance of our approach for data augmentation in a multilingual setting.

$\mathbf{O}_{\text {all }}+\mathbf{T r}_{\text {all } \rightarrow S} \quad$ We also experiment with translating the corpora from all the other languages into the source language to understand how the direction of the translation affects our approach and if our assumptions about translation direction are valid.

$\mathbf{O}_{\text {all }}+\operatorname{Tr}_{S \leftrightarrow \text { all }}$ This final configuration takes a concatenation of the original data in all languages with all the translated data obtained from both translation directions.

\begin{tabular}{lcccc}
\hline & \multicolumn{2}{c}{ Original } & \multicolumn{2}{c}{ Translated } \\
\hline Config. & Target & Others & $\mathbf{S} \rightarrow$ all & all $\rightarrow \mathbf{S}$ \\
\hline $\mathbf{O}_{T}$ (Monolingual) & $\checkmark$ & & & \\
$\mathbf{O}_{\text {all }}$ (Multilingual) & $\checkmark$ & $\checkmark$ & & \\
$\mathbf{O}_{\text {all }}+\mathbf{T r}_{S \rightarrow \text { all }}$ & $\checkmark$ & $\checkmark$ & $\checkmark$ & \\
$\mathbf{O}_{\text {all }}+\mathbf{T r}_{\text {all } \rightarrow S}$ & $\checkmark$ & $\checkmark$ & & $\checkmark$ \\
$\mathbf{O}_{\text {all }}+\mathbf{T r}_{S \leftrightarrow \text { all }}$ & $\checkmark$ & $\checkmark$ & $\checkmark$ & $\checkmark$ \\
\hline
\end{tabular}

TABLE 2: Data configurations for data augmentation.

\section{Experiments}

In this section, we describe the experiments that we conducted to explore the relevance of translation-based adaptation, both for cross-lingual adaptation, where annotated data is available in only one language, and for data augmentation, where multilingual datasets are available and we seek to improve performance from fine-tuning on the plain concatenation of data in all languages.

\subsection{Aspect-based Sentiment Analysis}

Aspect-based sentiment analysis (ABSA) may refer to several subtasks. We focus on joint opinion target extraction (OTE) and aspect sentiment classification (ASC). Following (Li et al., 2019a), we use a unified tagset with a single sequence tagging 


\begin{tabular}{c|cc|cc|cc|ccc|cc}
\hline & \multicolumn{4}{c}{ SemEval } \\
\hline \multirow{2}{*}{ Language } & \multicolumn{2}{c}{ Train } & \multicolumn{2}{c}{ Test } & \multicolumn{2}{c}{ Train } & \multicolumn{2}{c}{ Dev } & \multicolumn{2}{c}{ Test } \\
\cline { 2 - 11 } & \# review & \# OT & \# review & \# OT & \# review & \# OT & \# review & \# OT & \# review & \# OT \\
\hline ar & 4802 & 9612 & 1227 & 2371 & 592 & 741 & 198 & 280 & 198 & 237 \\
en & 2000 & 1743 & 676 & 612 & 600 & 780 & 200 & 279 & 200 & 269 \\
es & 2070 & 1859 & 881 & 713 & 1200 & 1080 & 200 & 354 & 200 & 372 \\
fr & 1664 & 1641 & 668 & 650 & 2047 & 1984 & 131 & 84 & 131 & 84 \\
nl & 1722 & 1859 & 575 & 713 & 594 & 554 & 198 & 183 & 198 & 190 \\
pl & - & - & - & - & 864 & 1827 & 200 & 610 & 200 & 581 \\
ro & - & - & - & - & 600 & 1050 & 200 & 358 & 200 & 357 \\
ru & 3665 & 3078 & 1209 & 952 & - & - & - & - & - & - \\
\hline
\end{tabular}

TABLE 3: Number of reviews for each language and corresponding number of Opinion Targets (OT).

model to jointly extract opinion targets and their associated sentiment label.

\subsection{Data}

We conducted experiments on common benchmark datasets for ABSA, as well as on in-house datasets annotated in the same way.

SemEval-2016 Task 5 (Pontiki et al., 2016) This shared task provides multilingual benchmark datasets for ABSA. Online reviews from several domains have been annotated for various languages. Datasets for OTE are available in English, Spanish, French, Dutch, Russian, Arabic, and Turkish. ${ }^{4}$ The annotations include opinion targets on which the opinion is voiced, along with their associated sentiment and the aspect category they belong to. The Arabic dataset pertains to the domain of hotel reviews, while the datasets in all the other languages contain restaurant reviews.

Orange In-house datasets To provide a different view of the same task, we experiment with in-house datasets annotated in a similar fashion. These datasets contain customer reviews for mobile applications. As such they contain noisy text which pose an additional challenge. The mobile applications are related to the activities of the Orange telco operator. Orange being present in several countries, we were able to gather and annotate reviews related to similar apps deployed in several countries. Our corpus covers French, English, Spanish, Arabic, ${ }^{5}$

4. We do not include the Turkish data in our experiments as no English to Turkish MT model is available within the NMT framework we use.

5. The application is deployed in several arabo-speaking countries, and the Arabic dataset includes standard Arabic along with 4 dialects (from Morocco, Tunisia, Jordan and Egypt). We are aware that this is a very rough approximation to launch a translation process independently of the dialect but we will try in future work to have more accurate translation systems for the corresponding dialects.
Cool app, I really like the ux design. Keep up !

Reallu useful app to know ur credit, internet consumption... Etc thnx

nice app all in hand to discover your account

Now it work! Except Invoice......Maybe because it's the first one.....For the rest no bad.

TABLE 4: Examples of reviews from the English inhouse dataset. Positive and negative opinion targets are shown in blue and red respectively.

Dutch, Polish, and Romanian. Examples of reviews in English are shown in Table 4.

Besides the difference in terms of domain, SemEval and in-house datasets differ in the level of segmentation. For SemEval, reviews were segmented into sentences and an input for the model is a single sentence, while for our dataset we kept reviews as a whole (sentence segmentation is not always present nor reliable and we considered it was more related to a real usage). As a consequence, inputs are longer for in-house dataset (for instance 22.8 words on average for English vs 14.1 for English SemEval or 34.8 words on average for Spanish vs 19.6 for Spanish SemEval). Moreover, the mobile applications reviews collected for the in-house datasets are directly typed through a mobile smartphone. As a result, data are more noisy in terms of typographic errors (see for instance the second example in Table 4).

\subsection{Source and Target Languages}

For the SemEval datasets, we translate the English corpus into all the other languages. Similarly, we use French as the source language for the in-house data. The choice of which language to translate from is based on the following considera- 


\begin{tabular}{l|cccccccccr}
\hline Word alignments & & ar & en & es & fr & nl & pl & ro & avg. & \% out \\
\hline \multirow{4}{*}{ fast_align } & no filtering & 24.9 & 38.7 & 31.7 & 50.6 & 53.8 & 22.0 & 19.8 & 34.5 & 0 \\
& $\alpha=2$ & 28.0 & 41.8 & 37.8 & 54.1 & 55.7 & 31.0 & 28.6 & 39.6 & 12.9 \\
& $\alpha=1$ & 27.9 & 41.2 & 35.1 & 52.5 & $\mathbf{5 6 . 1}$ & 30.1 & 26.1 & 38.4 & 13.3 \\
& $\alpha=0$ & 28.9 & 40.9 & 34.3 & 52.7 & 54.9 & 29.3 & 25.6 & 38.1 & 15.0 \\
\hline \multirow{5}{*}{ SimAlign } & no filtering & 22.8 & 31.4 & 29.5 & 39.1 & 37.9 & 26.2 & 23.3 & 30.0 & 0 \\
& $\alpha=2$ & 32.3 & 43.1 & $\mathbf{3 8 . 8}$ & 52.4 & 51.1 & 36.9 & $\mathbf{3 2 . 1}$ & 41.0 & 4.8 \\
& $\alpha=1$ & 31.9 & 42.4 & 38.7 & 53.2 & 54.5 & $\mathbf{3 7 . 7}$ & 31.5 & $\mathbf{4 1 . 4}$ & 5.3 \\
& $\alpha=0$ & $\mathbf{3 3 . 3}$ & $\mathbf{4 3 . 4}$ & 38.7 & $\mathbf{5 4 . 5}$ & 49.8 & 35.8 & 30.9 & 40.9 & 11.4 \\
\hline
\end{tabular}

TABLE 5: Study of the impact of constraining the number of insertions within projected opinion targets with spanbased alignment. Results for the $\mathbf{O}_{S}+\mathbf{T r}_{S \rightarrow \text { all }}$ configuration on the in-house Test datasets.

tions : (i) We expect pre-trained translation models for high-resource languages such as English and French to be more accurate than for languages with fewer resources. (ii) We consider that annotations for English or French data in either case tend to be of higher quality as they will go through a more extensive review process. In the case of the in-house datasets, French data is also more represented.

\subsection{Experimental Settings}

We use mBERT as our multilingual language model initialized with pre-trained weights ${ }^{6}$ available from HuggingFace Transformers (Wolf et al., 2020). For each experiment, a model is fine-tuned with a linear layer for sequence tagging added on top of mBERT's architecture. We train each model for a maximum of 50 epochs using the Adam optimizer (Kingma and $\mathrm{Ba}, 2015$ ) with a learning rate set to $5 e^{-5}$. We use the BIOES encoding as our tagging scheme. The evaluation is performed on exact matches of span boundaries and labels. Results are averaged over three runs and are reported using the micro $F_{1}$ score. In this study, we did not seek to use the most accurate model for the task and voluntarily chose a simple architecture in order to focus on training data selection and preparation.

\subsection{Annotation Projection}

To establish what is the optimal value for $\alpha$ on our task, we conduct a preliminary extrinsic evaluation. Using the translations of the French inhouse dataset in the other languages, we create different collections of synthetic datasets by projecting the annotations with different values for $\alpha$. We also evaluate our projection method with no restriction on the number of insertions to assess the relevance of the filtering approach. We fine-tune these models in the cross-lingual adapta-

6. bert-base-multilingual-cased tion scenario with the $\mathbf{O}_{S}+\operatorname{Tr}_{S \rightarrow \text { all }}$ configuration. We also compare SimAlign with fast_align. The results are shown in Table 5. We observe a clear gain with our filtering method compared to allowing any insertion. We also obtain better results with SimAlign. Removing data where a gap is observed filters out more data when using fast_align. Similar experiments on SemEval not reported here yielded similar conclusions. Based on these results, SimAlign is used in all the following experiments and $\alpha$ is set to 1 .

\subsection{Cross-lingual Adaptation}

Results are shown in Table 6. We compare our method with the work of Li et al. (2020), even though we were not able to reproduce their results with Zero-shot. They use fast_align and similar span alignment but without any constraints on allowed gaps. Hence our projection approach differs from theirs in the alignment method (SimAlign vs. fast_align) and in the filtering process that we introduced. And their training approach consists in warming up the model's parameters using the translated data and then fine-tuning on the source language data only.

Overall, performances vary across languages, with lower results for SemEval in particular in the Zero-shot configuration for distant languages (Russian and Arabic). Translation-based adaptation configurations, namely $\mathbf{O}_{S}+\operatorname{Tr}_{S \rightarrow T}$ and $\mathbf{O}_{S}$ $+\operatorname{Tr}_{S \rightarrow \text { all }}$, provide a significant performance improvement compared to Zero-shot. In some cases, $\operatorname{Tr}_{S \rightarrow T}$ performs better than Zero-shot, but there is a clear benefit to using the original source language data as well. Results are particularly low for Arabic since the dataset pertains to another domain (restaurant vs. hotel reviews). For SemEval, the gains observed for Arabic despite the domain shift demonstrate the usefulness of fine-tuning on synthetic data in the target language even if the source 
language data is from a different domain.

In most cases, $\mathbf{O}_{S}+\mathbf{T r}_{S \rightarrow T}$ and $\mathbf{O}_{S}+\mathbf{T r}_{S \rightarrow \text { all }}$ models come relatively close. However, $\mathbf{O}_{S}+$ $\operatorname{Tr}_{S \rightarrow \text { all }}$ generally outperforms the other configurations. Moreover, it can be considered as superior as a unique model can be applied to all languages which is particularly relevant from an operational point of view, with only one model to maintain. While the results between the approach of Li et al. (2020) and ours are similar, our approach has the advantage of being conceptually simpler and easier to implement.

We also observe that with $\mathbf{O}_{S}+\mathbf{T r}_{S \rightarrow \text { all }}$, the translated data contribute to improved performances for the source language, i.e. English or French, highlighting the relevance of the translation-based adaptation method for data augmentation as well.

\subsection{Data Augmentation}

Results for data augmentation are shown in Table 7. When comparing the two non-augmented baselines, i.e. Monolingual and Multilingual, we observe a significant improvement when fine-tuning a model on the combination of all languages (on average, +11.7 points for SemEval and +16.6 points on the in-house dataset), highlighting the ability of mBERT for cross-lingual transfer learning.

For SemEval, augmenting the training corpus with translated data consistently provides an improvement over the $\mathbf{O}_{\text {all }}$ baseline. Using the translations of the non-English corpora into English $\left(\mathbf{O}_{\text {all }}\right.$ $\left.+\operatorname{Tr}_{a l l \rightarrow S}\right)$ is detrimental to the performance on the English test set, while other languages are not impacted as much by this translation direction. The reason could be that, similarly to the cross-lingual adaptation experiments, it is beneficial to use the translated data in the target language specifically. Overall, $\mathbf{O}_{\text {all }}+\mathbf{T r}_{S \rightarrow \text { all }}$ seems to be the most effective configuration for all languages.

Regarding the in-house datasets, the synthetic data is not as beneficial as in the case of SemEval. Results are comparable to $\mathbf{O}_{\text {all }}$ for all translation directions, and no data configuration stands out as most effective on average.

\section{Analysis}

In line with the results from the previous section, we conduct a refined analysis to shed some light on the impact of noisy text.

When applied to the SemEval datasets, data augmentation in the resource-rich setting is beneficial and yields a significant improvement in performance, especially for English. For the in-house datasets however, data augmentation does not affect the performances as much compared to the multilingual baseline. Our hypothesis is that the inhouse datasets are more noisy and contain a more specific lexicon, which is what could be perturbing the translations, and thus negatively impacting the rest of the pipeline.

As we did not have gold data to explicitly evaluate the orthographic or grammatical deviation rate, nor the translation quality, nor the alignment quality, we propose an approximation to quantify noise and compare SemEval and in-house datasets. We compute the out-of-dictionary (OOD) rate using reference dictionaries recommended with Hunspell ${ }^{7}$ and available from Firefox for each language. ${ }^{8}$ The OOD rate is computed as follows :

$$
\text { OOD rate }=\frac{O}{N} \times 100
$$

where $O$ is the number of OOD words and $N$ the number of tokens in the corpus. Table 8 provides the OOD rate for the training sets of each language that is common to both SemEval and in-house data. We observe that the OOD rates are around twice as

\footnotetext{
7. http://hunspell.github.io/

8. https://addons.mozilla.org/en-US/
} firefox/language-tools/

\begin{tabular}{|c|c|c|c|c|c|c|c|c|c|c|c|c|c|}
\hline \multirow[b]{2}{*}{ Config. } & \multicolumn{6}{|c|}{ SemEval } & \multicolumn{7}{|c|}{ In-house } \\
\hline & en & ar & es & fr & nl & ru & fr & ar & en & es & nl & pl & ro \\
\hline $\mathbf{O}_{S}$ (Zero-shot) & 62.7 & 13.2 & 51.2 & 35.1 & 38.2 & 34.9 & 48.6 & 13.5 & 23.9 & 24.8 & 33.8 & 19.3 & 15.4 \\
\hline $\operatorname{Tr}_{S \rightarrow T}$ & - & 7.6 & 25.8 & 37.6 & 24.0 & 29.1 & - & 24.5 & 30.3 & 31.5 & 38.8 & 31.9 & 27.2 \\
\hline $\mathbf{O}_{S}+\mathbf{T r}_{S \rightarrow T}$ & & 31.4 & 54.3 & 40.7 & 49.4 & 47.7 & & 30.0 & 42.6 & 38.7 & 55.6 & 34.2 & 29.9 \\
\hline $\mathbf{O}_{S}+\mathbf{T r}_{S \rightarrow \text { all }}$ & 64.1 & 34.7 & 54.8 & 41.49 & 51.0 & 47.9 & 51.4 & 31.3 & 43.3 & 39.4 & 55.5 & 35.6 & 31.9 \\
\hline (Li et al., 2020) & - & - & 58.2 & 46.9 & 49.9 & 44.9 & - & - & - & - & - & - & - \\
\hline
\end{tabular}

TABLE 6: Results for cross-lingual adaptation, i.e. annotated data is available for the source language only. 


\begin{tabular}{|c|c|c|c|c|c|c|c|c|c|c|c|c|c|c|c|}
\hline \multirow[b]{2}{*}{ Config. } & \multicolumn{7}{|c|}{ SemEval } & \multicolumn{8}{|c|}{ In-house } \\
\hline & ar & en & es & fr & nl & ru & avg. & ar & en & es & $\mathbf{f r}$ & nl & pl & ro & avg. \\
\hline $\mathbf{O}_{T}$ (Monolingual) & 61.1 & 62.7 & 58.9 & 34.4 & 52.3 & 35.5 & 50.8 & 10.3 & 42.6 & 38.7 & 48.6 & 55.6 & 34.2 & 29.9 & 37.1 \\
\hline $\mathbf{O}_{\text {all }}$ (Multilingual) & 61.7 & 63.0 & 67.0 & 62.1 & 59.3 & 61.6 & 62.5 & 48.0 & 54.0 & 54.8 & 56.8 & 64.5 & 56.4 & 41.3 & 53.7 \\
\hline $\mathbf{O}_{\text {all }}+\mathbf{T r}_{S \rightarrow \text { all }}$ & 61.7 & 66.8 & 67.7 & 61.3 & 62.0 & 62.2 & 63.6 & 48.5 & 53.2 & 56.0 & 55.9 & 61.6 & 54.5 & 41.0 & 53.0 \\
\hline $\mathbf{O}_{a l l}+\mathbf{T r}_{a l l \rightarrow S}$ & 61.7 & 56.6 & 68.8 & 61.8 & 59.5 & 61.3 & 61.6 & 46.9 & 54.1 & 56.2 & 54.1 & 64.1 & 55.3 & 40.8 & 53.1 \\
\hline $\mathbf{O}_{\text {all }}+\mathbf{T r}_{S \leftrightarrow a l l}$ & 61.6 & 58.7 & 67.5 & 62.3 & 62.1 & 61.7 & 62.3 & 48.4 & 54.9 & 54.2 & 55.2 & 64.6 & 54.6 & 42.0 & 53.4 \\
\hline
\end{tabular}

TABLE 7: Results for data augmentation, i.e. annotated data is available for all languages.

high for our in-house datasets compared to SemEval datasets. ${ }^{9}$

\begin{tabular}{lllllll}
\hline & fr & en & nl & es & ar & avg. \\
\hline SemEval & 1.4 & 2.5 & 2.1 & 4.0 & 12.1 & 4.4 \\
In-house & 4.3 & 3.7 & 4.1 & 7.0 & 25.6 & 8.9 \\
\hline
\end{tabular}

TABLE 8: Average OOD rates in the training sets.

Considering the OOD rate as an indicator of noise in text, we can filter out the most noisy utterances from the source dataset in French before translating it into the other languages. We compute the OOD rate for each utterance and rank them from most noisy to least noisy. The top $n$ percent is then filtered out from the dataset before translation. We experiment with several values for $n$. Results can be seen in Table 9. We observe a slight increase in performance when filtering out the 5\% most noisy utterances compared to not filtering. Beyond that, filtering a larger portion of utterances seems to be reducing the size of the dataset too much and degrades performance.

\begin{tabular}{lllllll|l}
\hline Filter & ar & en & es & nl & pl & ro & avg. \\
\hline None & $\mathbf{4 8 . 5}$ & 53.2 & $\mathbf{5 6 . 0}$ & 61.6 & 54.5 & 41.0 & 52.5 \\
$\mathbf{5 \%}$ & 47.4 & $\mathbf{5 5 . 3}$ & 55.6 & $\mathbf{6 4 . 0}$ & 54.7 & $\mathbf{4 1 . 7}$ & $\mathbf{5 3 . 1}$ \\
$\mathbf{1 0 \%}$ & 46.8 & 53.2 & 54.5 & 63.3 & $\mathbf{5 4 . 8}$ & 40.9 & 52.2 \\
$\mathbf{2 0 \%}$ & 47.4 & 54.5 & 52.6 & 63.8 & 53.1 & 38.3 & 51.6 \\
\hline
\end{tabular}

TABLE 9: Filtering out the $n$ percent utterances with the largest OOD rate from the Source corpus before translating it into the other languages, impact on Target languages (data augmentation on the in-house dataset with the $\mathbf{O}_{\text {all }}+\mathbf{T r}_{S \rightarrow \text { all }}$ configuration)

\section{Conclusion}

We have proposed an efficient yet simple way of generating training data for a sequence tagging task based on translation and label projection. When applied to noisy data such as customer reviews, we

9. Note that the higher average OOD rate in Arabic can be attributed to dialectal variations. propose a way to overcome potentially ill-formed projections by filtering out some translated data thanks to a heuristic. Our SpanAlign algorithm, in conjunction with the SimAlign word alignment approach, yields interesting results for multilingual opinion mining both in cross-lingual configuration where no annotated data is available in the target languages, and in data augmentation configuration where annotated data are available in the target languages. We show that it is possible to train a single model for several languages, which is important in an industrial setting for maintenance issues. Finally, a preliminary study shows that selecting data prior to the translation and projection process on the basis of an Out of Dictionary rate increases the process robustness. Future work will consist in characterizing more precisely the level of noise in data and potentially correcting data in order to improve the selection process.

\section{References}

Carmen Banea, Rada Mihalcea, Janyce Wiebe, and Samer Hassan. 2008. Multilingual subjectivity analysis using machine translation. In Proceedings of the 2008 Conference on Empirical Methods in Natural Language Processing, pages 127-135, Honolulu, Hawaii. Association for Computational Linguistics.

Yun Chen, Yang Liu, Guanhua Chen, Xin Jiang, and Qun Liu. 2020. Accurate word alignment induction from neural machine translation. In Proceedings of the 2020 Conference on Empirical Methods in Natural Language Processing (EMNLP), pages 566-576, Online. Association for Computational Linguistics.

Alexis Conneau, Kartikay Khandelwal, Naman Goyal, Vishrav Chaudhary, Guillaume Wenzek, Francisco Guzmán, Edouard Grave, Myle Ott, Luke Zettlemoyer, and Veselin Stoyanov. 2020. Unsupervised cross-lingual representation learning at scale. In Proceedings of the 58th Annual Meeting of the Association for Computational Linguistics, pages 8440 8451, Online. Association for Computational Linguistics.

Alexis Conneau, Ruty Rinott, Guillaume Lample, Adina Williams, Samuel R. Bowman, Holger Schwenk, and Veselin Stoyanov. 2018. Xnli : Evaluating cross-lingual sentence representations. In 
Proceedings of the 2018 Conference on Empirical Methods in Natural Language Processing. Association for Computational Linguistics.

Kevin Duh, Akinori Fujino, and Masaaki Nagata. 2011 Is machine translation ripe for cross-lingual sentiment classification? In Proceedings of the 49th Annual Meeting of the Association for Computational Linguistics : Human Language Technologies, pages 429-433, Portland, Oregon, USA. Association for Computational Linguistics.

Chris Dyer, Victor Chahuneau, and Noah A. Smith. 2013. A simple, fast, and effective reparameterization of IBM model 2. In Proceedings of the 2013 Conference of the North American Chapter of the Association for Computational Linguistics : Human Language Technologies, pages 644-648, Atlanta, Georgia. Association for Computational Linguistics.

Zhifang Fan, Zhen Wu, Xin-Yu Dai, Shujian Huang, and Jiajun Chen. 2019. Target-oriented opinion words extraction with target-fused neural sequence labeling. In Proceedings of the 2019 Conference of the North American Chapter of the Association for Computational Linguistics : Human Language Technologies, Volume 1 (Long and Short Papers). Association for Computational Linguistics.

Hao Fei, Meishan Zhang, and Donghong Ji. 2020. Cross-lingual semantic role labeling with highquality translated training corpus. In Proceedings of the 58th Annual Meeting of the Association for Computational Linguistics, pages 7014-7026.

Minqing $\mathrm{Hu}$ and Bing Liu. 2004. Mining and summarizing customer reviews. In Proceedings of the Tenth ACM SIGKDD International Conference on Knowledge Discovery and Data Mining, KDD '04, page 168-177, New York, NY, USA. Association for Computing Machinery.

Alankar Jain, Bhargavi Paranjape, and Zachary C. Lipton. 2019. Entity projection via machine translation for cross-lingual ner. In Proceedings of the 2019 Conference on Empirical Methods in Natural Language Processing and the 9th International Joint Conference on Natural Language Processing, EMNLP-IJCNLP 2019, Hong Kong, China, November 3-7, 2019, pages 1083-1092. Association for Computational Linguistics.

Masoud Jalili Sabet, Philipp Dufter, François Yvon, and Hinrich Schütze. 2020. SimAlign: High quality word alignments without parallel training data using static and contextualized embeddings. In Proceedings of the 2020 Conference on Empirical Methods in Natural Language Processing : Findings, pages 1627-1643, Online. Association for Computational Linguistics.

Soufian Jebbara and Philipp Cimiano. 2019. Zero-shot cross-lingual opinion target extraction. In Proceedings of the 2019 Conference of the North American Chapter of the Association for Computational Linguistics : Human Language Technologies, Volume 1 (Long and Short Papers), pages 2486-2495, Minneapolis, Minnesota. Association for Computational Linguistics.
Diederik P. Kingma and Jimmy Ba. 2015. Adam: A method for stochastic optimization. In $3 \mathrm{rd}$ International Conference on Learning Representations, ICLR 2015, San Diego, CA, USA, May 7-9, 2015, Conference Track Proceedings.

X. Li, Lidong Bing, Wenxuan Zhang, Zheng Li, and Wai Lam. 2020. Unsupervised cross-lingual adaptation for sequence tagging and beyond. ArXiv, $\mathrm{abs} / 2010.12405$.

Xin Li, Lidong Bing, Piji Li, and Wai Lam. 2019a. A unified model for opinion target extraction and target sentiment prediction. Proceedings of the AAAI Conference on Artificial Intelligence, 33 :67146721.

Xin Li, Lidong Bing, Wenxuan Zhang, and W. Lam. 2019b. Exploiting bert for end-to-end aspect-based sentiment analysis. ArXiv, abs/1910.00883.

Xin Li and Wai Lam. 2017. Deep multi-task learning for aspect term extraction with memory interaction. In Proceedings of the 2017 Conference on Empirical Methods in Natural Language Processing, pages 2886-2892, Copenhagen, Denmark. Association for Computational Linguistics.

Gabriel Marzinotto. 2020. FrameNet annotations alignment using attention-based machine translation. In Proceedings of the International FrameNet Workshop 2020 : Towards a Global, Multilingual FrameNet, pages 41-47, Marseille, France. European Language Resources Association.

Bo Pang and Lillian Lee. 2008. Opinion mining and sentiment analysis. Found. Trends Inf. Retr. $2(1-2): 1-135$.

Haiyun Peng, Lu Xu, Lidong Bing, Fei Huang, Wei Lu, and Luo Si. 2020. Knowing what, how and why: A near complete solution for aspect-based sentiment analysis. Proceedings of the AAAI Conference on Artificial Intelligence, 34(05) :8600-8607.

Telmo Pires, Eva Schlinger, and Dan Garrette. 2019. How multilingual is multilingual BERT? In Proceedings of the 57th Annual Meeting of the Association for Computational Linguistics, pages 49965001, Florence, Italy. Association for Computational Linguistics.

Maria Pontiki, Dimitris Galanis, Haris Papageorgiou, Ion Androutsopoulos, Suresh Manandhar, Mohammad AL-Smadi, Mahmoud Al-Ayyoub, Yanyan Zhao, Bing Qin, Orphée De Clercq, Véronique Hoste, Marianna Apidianaki, Xavier Tannier, Natalia Loukachevitch, Evgeniy Kotelnikov, Nuria Bel, Salud María Jiménez-Zafra, and Gülşen Eryiğit. 2016. SemEval-2016 task 5: Aspect based sentiment analysis. In Proceedings of the 10th International Workshop on Semantic Evaluation (SemEval2016), pages 19-30, San Diego, California. Association for Computational Linguistics.

Maria Pontiki, Dimitris Galanis, Haris Papageorgiou, Suresh Manandhar, and Ion Androutsopoulos. 2015. SemEval-2015 task 12: Aspect based sentiment analysis. In Proceedings of the 9th International Workshop on Semantic Evaluation (SemEval 2015), pages 
486-495, Denver, Colorado. Association for Computational Linguistics.

Maria Pontiki, Dimitris Galanis, John Pavlopoulos, Harris Papageorgiou, Ion Androutsopoulos, and Suresh Manandhar. 2014. SemEval-2014 task 4: Aspect based sentiment analysis. In Proceedings of the 8th International Workshop on Semantic Evaluation (SemEval 2014), pages 27-35, Dublin, Ireland. Association for Computational Linguistics.

Ana-Maria Popescu and Oren Etzioni. 2005. Extracting product features and opinions from reviews. In Proceedings of the Conference on Human Language Technology and Empirical Methods in Natural Language Processing, HLT '05, page 339-346, USA. Association for Computational Linguistics.

Amir Pouran Ben Veyseh, Nasim Nouri, Franck Dernoncourt, Dejing Dou, and Thien Huu Nguyen. 2020. Introducing syntactic structures into target opinion word extraction with deep learning. In Proceedings of the 2020 Conference on Empirical Methods in Natural Language Processing (EMNLP), pages 8947-8956, Online. Association for Computational Linguistics.

Alec Radford, Jeff Wu, R. Child, David Luan, Dario Amodei, and Ilya Sutskever. 2019. Language models are unsupervised multitask learners. In OpenAI blog.

Holger Schwenk and Xian Li. 2018. A corpus for multilingual document classification in eight languages. In Proceedings of the Eleventh International Conference on Language Resources and Evaluation (LREC 2018). European Language Resources Association (ELRA).

Jörg Tiedemann and Santhosh Thottingal. 2020. OPUS-MT — Building open translation services for the World. In Proceedings of the 22nd Annual Conferenec of the European Association for $\mathrm{Ma}$ chine Translation (EAMT), Lisbon, Portugal.

Fang Wang, Yuncong Li, Wenjun Zhang, and Shengtao Zhong. 2021. A more fine-grained aspectsentiment-opinion triplet extraction task. ArXiv, abs/2103.15255.

Thomas Wolf, Lysandre Debut, Victor Sanh, Julien Chaumond, Clement Delangue, Anthony Moi, Pierric Cistac, Tim Rault, Remi Louf, Morgan Funtowicz, Joe Davison, Sam Shleifer, Patrick von Platen, Clara Ma, Yacine Jernite, Julien Plu, Canwen Xu, Teven Le Scao, Sylvain Gugger, Mariama Drame, Quentin Lhoest, and Alexander Rush. 2020. Transformers: State-of-the-art natural language processing. In Proceedings of the 2020 Conference on Empirical Methods in Natural Language Processing : System Demonstrations, pages 38-45, Online. Association for Computational Linguistics.

Shijie Wu and Mark Dredze. 2019. Beto, bentz, becas: The surprising cross-lingual effectiveness of BERT. In Proceedings of the 2019 Conference on Empirical Methods in Natural Language Processing and the 9th International Joint Conference on Natural Language Processing (EMNLP-IJCNLP), pages 833-844, Hong Kong, China. Association for Computational Linguistics.
$\mathrm{Hu}$ Xu, Bing Liu, Lei Shu, and Philip S. Yu. 2018. Double embeddings and cnn-based sequence labeling for aspect extraction. Proceedings of the 56th Annual Meeting of the Association for Computational Linguistics (Volume 2 : Short Papers).

Weijia Xu, Batool Haider, and Saab Mansour. 2020. End-to-end slot alignment and recognition for crosslingual NLU. In Proceedings of the 2020 Conference on Empirical Methods in Natural Language Processing (EMNLP), pages 5052-5063, Online. Association for Computational Linguistics.

Yinfei Yang, Yuan Zhang, Chris Tar, and Jason Baldridge. 2019. PAWS-X : A cross-lingual adversarial dataset for paraphrase identification. In Proceedings of the 2019 Conference on Empirical Methods in Natural Language Processing and the 9th International Joint Conference on Natural Language Processing (EMNLP-IJCNLP), pages 3687-3692. Association for Computational Linguistics.

Vilém Zouhar and Daria Pylypenko. 2021. Leveraging neural machine translation for word alignment. 